\title{
Gender Differences in German Upward Income Mobility
}

\author{
Ira N. Gang \\ Rutgers University \\ gang@economics.rutgers.edu \\ John Landon-Lane \\ Rutgers University \\ lane@economics.rutgers.edu \\ Myeong-Su Yun* \\ Tulane University \\ msyun@tulane.edu
}

August 19, 2002

\begin{abstract}
We examine the upward labor income mobility of men and women in Germany using the GSOEP Cross National Equivalent File. Women have greater overall income mobility. However, utilizing a measure of upward income mobility and calculating the posterior probability that men's upward income mobility is greater than women's, we find that men have overall greater upward income mobility. Women have greater upward mobility in the lower intial income classes, in the upper initial income brackets men's mobility is higher than women's.
\end{abstract}

Keywords: upper income mobility, Markov chain, income distribution dynamics, gender discrimination

JEL Classification: D3, D63, J7

${ }^{*}$ Corresponding Author: John Landon-Lane, Economics Department, NJH, Rutgers University, 75 Hamilton St, New Brunswick NJ 08901-1248. An earlier version of this paper was presented at the 5th International German Socio-Economic Panel User Conference, July 3-4, 2002, Berlin, Germany. 


\section{Introduction}

In this paper we explore the upward income mobility of men and women in Germany over the period 1984 to 1997. In terms of labor income mobility we examine whether men and women have approximately the same degree of upward mobility across the income distribution and whether upward income mobility varies by gender among the lower, middle and upper parts of the distribution.

We examine the labor income mobility of men and women in Germany using the GSOEP Cross National Equivalent File. We examine the dynamics of the income distribution - the movement of women and men through the distribution of income over time. We model the dynamics of the income distribution as a first order Markov chain. Bayesian methods are used to characterize the distribution of all the functions of the transition probability matrix. In particular, we are able to estimate the probabilities of an individual moving from one income classification to another, formally compare and contrast various mobility indices across different subsamples of the data, and formally compare and test various hypotheses on the convergence properties of the income distribution. We are most interested in developing measures of upward income mobility and testing different hypotheses on the transitional dynamics of the income distribution.

In the next section we discuss various income mobility measures and characterize our upward mobility measures. Section 3 discusses the data and our priors. Results are discussed in section 4 . Section 5 concludes.

\section{Measuring Upward Income Mobility}

In this paper we apply the results from Gang, Landon-Lane and Yun (2002b) to data from Germany. In what follows is a brief discussion of the model and the estimation strategy. We model the dynamics of labor income using a first order Markov chain. The 
use of Markov-chain models to study income dynamics has a long history with notable contributions by Champernowne (1953) and Shorrocks (1976).

Using the Markov assumption there are many measures of overall income mobility that one may define. For complete discussions of the properties and definitions of a large number of mobility measures see Shorrocks (1978) and Geweke, Marshall and Zarkin (1986). A measure of overall income mobility measure that is commonly reported in the literature is the measure due to Shorrocks (1978), which is defined as

$$
\mathcal{M}_{s}(\mathbf{P})=\frac{C-\operatorname{tr}(\mathbf{P})}{C-1}
$$

There are $C$ income classifications where $C$ is a finite number. The probability of transiting from class $i$ in period $t-1\left(\pi_{t-1}=i\right)$ to class $j$ in period $t\left(\pi_{t}=j\right)$ is $P\left(\pi_{t}=j \mid \pi_{t-1}=i\right) \equiv p_{i j}$, so that the Markov transition matrix, $\mathbf{P}$, can be defined as $\mathbf{P}=\left[p_{i j}\right]$.

Note that we assume that the transition probability follows the first order Markov chain property, that is,

$$
P\left(\pi_{t} \mid \pi_{t-1}, \pi_{t-2}, \ldots, \pi_{t-j}\right)=P\left(\pi_{t} \mid \pi_{t-1}\right) \quad \forall j=2,3, \ldots,
$$

where $P($.$) represents the conditional probability distribution of \pi$. The first order Markov property implies that $\pi_{t}^{\prime}=\pi_{0}^{\prime} \mathbf{P}^{t}$, where $\pi_{0}$ is the initial income distribution. The invariant or limiting income distribution, $\bar{\pi}$, is any distribution that satisfies

$$
\bar{\pi}^{\prime}=\bar{\pi}^{\prime} \mathbf{P}
$$

The invariant distribution is unique if there is only one eigenvalue of $\mathbf{P}$ with modulus one. $^{1}$

\footnotetext{
${ }^{1}$ Implicitly we are assuming that the eigenvalues have been ordered from highest to lowest in terms of magnitude. As $\mathbf{P}$ is row stochastic we know that the highest eigenvalue, in terms of magnitude, is
} 
The Shorrocks measure can be shown to be the inverse of the harmonic mean of the expected length of stay in an income class, scaled by a factor of $C /(C-1)$. This index satisfies the monotonicity, immobility and strong immobility persistence criteria and hence is internally consistent. ${ }^{2}$

In Gang, Landon-Lane, and Yun (2002b), we show how this measure can be decomposed into its upward and downward income mobility components. We also show that these upward and downward income mobility indices are internally consistent with respect to the persistence criteria noted above.

The measure of upward mobility that we use is

$$
\overline{\mathcal{M}}_{U \mid i}(\mathbf{P})=\frac{1}{C-1} \sum_{k=1}^{C-1} \mathcal{M}_{U \mid k}(\mathbf{P})
$$

where

$$
\mathcal{M}_{U \mid i}(\mathbf{P})=\sum_{k=i+1}^{C} p_{i k}
$$

Here, $\mathcal{M}_{U \mid i}$ measures the conditional probability of moving up from income class $i$ to an income class above $i$, and $\overline{\mathcal{M}}_{U \mid i}$ is the average conditional probability of moving to a higher income class. These measures allow us to characterize any differences between males and females in terms of ability of moving to a higher income class.

The mobility measures estimated using a Bayesian methods will be reported in section 4 . In Section 4 we also report the posterior probability that the upward income mobility for males is higher than the upward income mobility for females.

1. If the magnitude of the second eigenvalue is strictly less than 1 then we know that the invariant distribution is unique.

${ }^{2}$ See Geweke, Marshall, and Zarkin (1986) for a complete discussion on the properties of these mobility indices. 


\section{Data and Prior Distribution}

\subsection{Data}

We need panel data in order to study gender differences in upward mobility in labor income. We use samples drawn from the German Socio-Economic Panel (GSOEP) from Germany. The study is further facilitated by using GSEOP files from the Cross National Equivalent File (CNEF), which standardizes the information in the GSOEP. ${ }^{3}$ The GSOEP-CNEF contains information regarding not only demographic characteristics but also labor market activities including labor income. Our variable of interest is real annual labor income. ${ }^{4}$

We utilize the West German sample from the GSOEP (sample A). We also exclude those who work in agriculture. In order to study only workers who have strong attachment to the labor market, we restrict the sample to those who work in full-time jobs in both starting and ending years, 1984 and $1997 .{ }^{5}$ Full-time workers are those who work 35 hours or more per week on average. We study only workers not younger than 25 years in the beginning year and not older than 60 years in the ending year of the period. For example, we select people from age 25 to 47 in 1984 when we study the 13 year transition between 1984 and 1997.

Table 1 shows labor income and Germany for 1984 and 1997. To gain some perspective on the sample we use in our analysis, Table 1 shows the incomes and sample sizes of all workers (including part-time), full time workers in the unbalanced panel, and full time

\footnotetext{
${ }^{3}$ The GSOEP-CNEF are available thanks to efforts of researchers and staff at Cornell University and the German Institute for Economic Research (DIW). For details of making equivalent files across countries, see the homepage of this project, http://www.human.cornell.edu/pam/gsoep/equivfil.cfm.

${ }^{4}$ We compute labor income using the consumer price index (base year: 1991) and converting German Marks to US dollars using a purchasing power parity exchange rate (PPP) in 1991. The PPP in 1991 is $2.09 \mathrm{DM}$ per one US dollar, while the exchange rate in the same year is $1.66 \mathrm{DM}$ per a dollar. Also note that we rescaled German CPI by moving the base year to 1991 from 1999. This was done in preparation for other comparative work in which we are engaged. See Gang, Landon-Lane and Yun (2002a).

${ }^{5}$ We choose people who were full-time job workers in both year 1984 and 1997 and study study the 13 year transition. The fact that they worked in full-time jobs in both years does not necessarily mean that they worked in a full-time job throughout the period.
} 
workers appearing in both the 1984 and 1997 samples. The sample we are using, "careerworkers", i.e., those who are full time workers both in the beginning and ending periods, have the highest incomes. In our sample, German men in 1984 enjoy an annual labor income premium of 46.71 percent over women. For 1997 this premium is 31.38 percent.

Table 1: Mean Income Level (constant US\$, base year = 1991)

\begin{tabular}{|c|c|c|c|c|}
\hline & \multicolumn{4}{|c|}{ Germany } \\
\hline & \multicolumn{2}{|c|}{1984} & \multicolumn{2}{|c|}{1997} \\
\hline & Male & Female & Male & Female \\
\hline \multirow{4}{*}{$\begin{array}{l}\text { mean } \\
\text { std. dev. } \\
\text { sample size }\end{array}$} & \multicolumn{4}{|c|}{ Full-time workers in both years } \\
\hline & 26553 & 18099 & 32377 & 24644 \\
\hline & $(23694)$ & $(8214)$ & $(16569)$ & $(10051)$ \\
\hline & 643 & 132 & 643 & 132 \\
\hline \multicolumn{5}{|c|}{ Full-time workers in respective year } \\
\hline mean & 25507 & 17028 & 32618 & 22952 \\
\hline std. dev. & $(21857)$ & $(12012)$ & $(16565)$ & $(12396)$ \\
\hline sample size & 1480 & 503 & 748 & 241 \\
\hline & \multicolumn{4}{|c|}{ Workers in respective year } \\
\hline mean & 24276 & 12198 & 31363 & 15716 \\
\hline std. dev. & $(21450)$ & $(10367)$ & $(16546)$ & $(11891)$ \\
\hline sample size & 1657 & 1035 & 824 & 566 \\
\hline
\end{tabular}

Note1: Workers are restricted to working in non-agriculture and aged 25 to 47 years old in 1984 . Note2: German Mark is converted to U.S. dollar using PPP in 1991 (2.09DM/US\$)

\subsection{Prior Distributions}

This paper uses Bayesian methods to estimate and make inferences from the Markov chain model outlined in section 2. One important consequence of using Bayesian methods is that it is simple to characterize the distribution of any function of the primal parameters, $\pi_{0}$ and $\mathbf{P}$, of the model and any, possibly non-linear, function of these primal parameters. In this paper the functions of the primal parameters that we are interested in are the various mobility measures described above. 
As we use a Bayesian estimation strategy we need to construct priors for the unknown parameters of our model. The unknown parameters of the first order Markov chain model are $\pi_{0}$ and $\mathbf{P}$. We propose conjugate Dirichlet priors for $\pi_{0}$ and $\mathbf{P}$ parameterized by the vector $a_{0}$ and the matrix $\mathbf{A}$ respectively. These priors have a notional data interpretation in that $a_{i 0}-1$ can be interpreted as the number of individuals initially contained in income class i, while $\mathbf{A}_{i j}-1$ can be interpreted as the number of individuals transiting from income class $\mathrm{i}$ to income class $\mathrm{j}$ in the notional prior data set.

We take a neutral stance with our priors in that we want the data to tell the story. Noting that the prior has a notional data interpretation, we propose priors that are generated from a notional data set that is one tenth the size of the observed sample. For example, if the sample that we are using contains one thousand individuals then the prior would be parameterized so that it could be interpreted as coming from a notional sample of 100 individuals.

The prior distributions for all data sets used in this paper are scalar multiples of the following prior distributions. Table 2 contains the values for $a_{0}$ while Table 3 contains the values for $\mathbf{A}$ assuming a notional sample size of 100. In this analysis we define ten income classes that are equal in log length, following Champernowne (1953).

\section{Table 2: Prior for Initial Distribution: $\pi_{0}$}

\begin{tabular}{ccccccccccc} 
Income Class & 1 & 2 & 3 & 4 & 5 & 6 & 7 & 8 & 9 & 10 \\
\hline$a_{0 i}$ & 11 & 11 & 11 & 11 & 11 & 11 & 11 & 11 & 11 & 11
\end{tabular}

We place a flat prior over the parameters of the initial distribution. That is, we assume that all individuals have an equal chance of initially being in any income class. The prior for $\mathbf{P}$ has the characteristic, in order to be consistent with $a_{0}$, that there are ten individuals initially in each income class. The matrix $\mathbf{A}$ is then designed so that the highest prior probability is given to an individual staying in the same income class that she started in with decreasing probability given to moves further away from the starting 
Table 3: Prior for Transition Matrix: $\mathbf{P}$

\begin{tabular}{ccccccccccc} 
Income Class & 1 & 2 & 3 & 4 & 5 & 6 & 7 & 8 & 9 & 10 \\
\hline$A_{1 i}$ & 6.21 & 3.60 & 2.30 & 1.65 & 1.13 & 1.06 & 1.01 & 1.01 & 1.01 & 1.01 \\
$A_{2 i}$ & 3.06 & 5.13 & 3.06 & 2.03 & 1.51 & 1.10 & 1.05 & 1.01 & 1.01 & 1.01 \\
$A_{3 i}$ & 1.93 & 2.87 & 4.75 & 2.87 & 1.93 & 1.46 & 1.09 & 1.04 & 1.01 & 1.01 \\
$A_{4 i}$ & 1.44 & 1.89 & 2.79 & 4.58 & 2.79 & 1.89 & 1.44 & 1.08 & 1.04 & 1.01 \\
$A_{5 i}$ & 1.08 & 1.44 & 1.88 & 2.77 & 4.55 & 2.77 & 1.88 & 1.44 & 1.08 & 1.04 \\
$A_{6 i}$ & 1.04 & 1.08 & 1.44 & 1.88 & 2.77 & 4.55 & 2.77 & 1.88 & 1.44 & 1.08 \\
$A_{7 i}$ & 1.01 & 1.04 & 1.08 & 1.44 & 1.89 & 2.79 & 4.58 & 2.79 & 1.89 & 1.44 \\
$A_{8 i}$ & 1.01 & 1.01 & 1.04 & 1.09 & 1.46 & 1.93 & 2.87 & 4.75 & 2.87 & 1.93 \\
$A_{9 i}$ & 1.01 & 1.01 & 1.01 & 1.05 & 1.10 & 1.51 & 2.03 & 3.06 & 5.13 & 3.06 \\
$A_{10 i}$ & 1.01 & 1.01 & 1.01 & 1.01 & 1.06 & 1.13 & 1.65 & 2.30 & 3.60 & 6.21
\end{tabular}

income class. This prior is symmetric in the sense that the decline in the prior transition probability is not dependent on whether the move was to a lower or higher income class. This prior is neutral in the sense that there is equal prior probability assigned to all individuals of attaining any income class in the invariant distribution.

\section{Results}

We report a Shorrocks measure of overall income mobility, $\mathcal{M}_{s}(\mathbf{P})$ (see (1)), which is an average, across all income classes, of the conditional probabilities of an individual moving out of their current income class. Note that this measure is a measure of upward and downward mobility combined. We also report our measure of upward mobility, $\overline{\mathcal{M}}_{U \mid i}$. We report both measures for the full sample and we report $\overline{\mathcal{M}}_{U \mid i}$ for low, middle and high sub-groups of the income classes.

Following Champernowne (1953), real incomes for Germany were divided up into ten income classes that are equal in log length. ${ }^{6}$ The income class definitions are given in Table 4 below, as are the low, middle and high income subgroups. A number of different

\footnotetext{
${ }^{6}$ The first and tenth income class were designed to contain the bottom five percent and top five percent of the income distribution respectively.
} 
models were estimated. When modelling income mobility there is always uncertainty over the appropriate definition of the transition period. In this paper we estimate a Markov chain model for a 13 year transition period, 1984 to $1997 .^{7}$ For this estimation, we include in our sample only those individuals, age from 25 to 47 in the initial year, which in this case is 1984, that were full time employees in both the initial period and the final period of the transition. One benefit of defining the transition period to be thirteen years is that there is enough time to allow workers to progress in their chosen careers, hence allowing for the greatest chance of a transition out of their initial income class. However, defining such a large transition period comes at a price of reducing the number of individuals that we observe.

Table 4: Income Class Definitions: 1991 US\$

\begin{tabular}{ccc} 
Income Class & Income Range & Sub-Group \\
\hline 1 & {$[0,10000)$} & Low \\
2 & {$[10000,12375)$} & Low \\
3 & {$[12375,15314)$} & Low \\
4 & {$[15314,18951)$} & Middle \\
5 & {$[18951,23452)$} & Middle \\
6 & {$[23452,29022)$} & Middle \\
7 & {$[29022,35915)$} & High \\
8 & {$[35915,44444)$} & High \\
9 & {$[44444,55000)$} & High \\
10 & {$[55000, \infty)$} & High
\end{tabular}

The posterior means and standard deviations for $\pi_{0}, \mathbf{P}$, and $\bar{\pi}$ for males and females are presented in Appendix tables A.1 and A.2. The estimates can be characterized in the following way: Males in Germany have an initial income distribution that has more weight in the upper five income classes than the corresponding initial distribution for females. Moreover, the estimated transitions matrices are such that the invariant distributions for

\footnotetext{
${ }^{7}$ In order to check the robustness of the results to the definition of the transition period we also estimate a Markov chain with a five year transition using data from the beginning, middle and end of the sample. For the five year transitions we use full-time non-agricultural workers between the ages of 25 and 55 in the initial year of the transition. Qualitatively, all of our results are robust to the choice of transition period.
} 
males, also have more mass in the upper income classes than the corresponding invariant distributions for females.

The mobility measures are presented in Table 5 .

Table 5: Mobility Measures for 13 year transition

Mobility Measure Transition Period Group Male Female Prob[Male $>$ Female]

\section{Germany}

\begin{tabular}{|c|c|c|c|c|c|}
\hline $\mathcal{M}_{s}(\mathbf{P})$ & 1984-1997 & All & $\begin{array}{c}0.918 \\
(0.019)\end{array}$ & $\begin{array}{c}0.940 \\
(0.028)\end{array}$ & 0.250 \\
\hline$\overline{\mathcal{M}}_{U \mid i}$ & 1984-1997 & All & $\begin{array}{c}0.655 \\
(0.021)\end{array}$ & $\begin{array}{c}0.584 \\
(0.029)\end{array}$ & 0.974 \\
\hline$\overline{\mathcal{M}}_{U \mid i}$ & 1984-1997 & Low & $\begin{array}{c}0.771 \\
(0.045)\end{array}$ & $\begin{array}{c}0.812 \\
(0.042)\end{array}$ & 0.253 \\
\hline$\overline{\mathcal{M}}_{U \mid i}$ & 1984-1997 & Middle & $\begin{array}{c}0.722 \\
(0.021)\end{array}$ & $\begin{array}{c}0.703 \\
(0.044)\end{array}$ & 0.641 \\
\hline$\overline{\mathcal{M}}_{U \mid i}$ & 1984-1997 & High & $\begin{array}{c}0.473 \\
(0.038)\end{array}$ & $\begin{array}{c}0.236 \\
(0.064)\end{array}$ & 0.998 \\
\hline
\end{tabular}

We see that females in Germany have greater income mobility overall. The posterior probability that the value of $\mathcal{M}_{s}(\mathbf{P})$ for males is higher than the corresponding values for females is 0.250 . This implies that females have more overall mobility than males. However, when we look at the conditional probability measures, a different story emerges. First, males in Germany have an higher average conditional probability of moving up to a higher income class, 0.655 for men, 0.584 for women, the posterior probability being 0.974 . When broken down over subclasses, we see that females have higher upward mobility in the lowest income group, Low, whereas males and females have similar upward income mobility in the middle income group, Middle. However, in the highest income group we see males totally dominating females in terms of the conditional probability of moving to a higher income class.

We find that while females have higher mobility than males, a quite different story appears when we look at upward income mobility. Overall men's upward mobility exceeds 
women's. Higher female mobility is constrained to the lower income classes. In the middle income brackets the story is mixed. Females have a significantly lower upward income mobility than males in the highest income classes.

\section{Conclusion}

In this paper, we examined the dynamics of the income distribution of a country as a finite state first order Markov chain, using German data. We estimated this model using Bayesian methods with a neutral prior that was designed to reflect relative uncertainty on behalf of the researcher. Once estimated we then were able to analyze the income mobility properties of the data. In particular, we analyzed the upward income mobility characteristics of the data with respect to males and females.

We studied where women and men are located in the labor income distribution and the change in this position over time. Our study of the labor income mobility of men and women in Germany employed the Cross National Equivalent File, drawn from the German Socio-Economic Panel.

Overall, while females in Germany enjoy greater overall income mobility, we find that males have a significantly higher upward income mobility for the higher initial income classes. Females' upward income mobility measures compare favorably with males in the lower income classes, with mixed results in the middle income classes. 


\section{References}

Champernowne, D. G. (1953). 'A model of income distribution.' Economic Journal 63(250), 318-351.

Gang, I. N., J. S. Landon-Lane, and M-S. Yun (2002a). 'Does the glass ceiling exist?: A cross-national perspective on gender income mobility.' mimeo.

_ (2002b). 'Measuring upward income mobility: Theory and applications.' mimeo.

Geweke, J. F., C. Marshall, and G. A. Zarkin (1986). 'Mobility indices in continuous time Markov chains.' Econometrica 54, 1407-1423.

Shorrocks, A. F. (1976). 'Income mobility and the Markov assumption.' Economic Journal 86, 566-578. (1978). 'The measurement of mobility.' Econometrica 46, 1013-1024. 


\section{A Posterior Estimates for German Data}

Table A.1: Posterior Estimates: GERMAN Males (1984-1997)

Initial Distribution: $\pi_{0}$

$\begin{array}{cccccccccc}0.023 & 0.022 & 0.070 & 0.201 & 0.233 & 0.197 & 0.130 & 0.055 & 0.031 & 0.038 \\ (0.006) & (0.006) & (0.009) & (0.015) & (0.016) & (0.015) & (0.012) & (0.009) & (0.007) & (0.007)\end{array}$

Transition Matrix : P

$\begin{array}{cccccccccc}0.172 & 0.106 & 0.072 & 0.136 & 0.080 & 0.161 & 0.077 & 0.078 & 0.040 & 0.079 \\ (0.075) & (0.059) & (0.052) & (0.068) & (0.052) & (0.071) & (0.050) & (0.051) & (0.039) & (0.049) \\ 0.176 & 0.150 & 0.094 & 0.110 & 0.139 & 0.087 & 0.042 & 0.121 & 0.042 & 0.040 \\ (0.077) & (0.072) & (0.060) & (0.062) & (0.071) & (0.056) & (0.040) & (0.063) & (0.040) & (0.040) \\ 0.061 & 0.053 & 0.073 & 0.207 & 0.260 & 0.141 & 0.103 & 0.051 & 0.033 & 0.018 \\ (0.031) & (0.029) & (0.033) & (0.052) & (0.057) & (0.044) & (0.040) & (0.029) & (0.023) & (0.017) \\ 0.021 & 0.023 & 0.027 & 0.137 & 0.271 & 0.323 & 0.140 & 0.039 & 0.013 & 0.007 \\ (0.011) & (0.011) & (0.013) & (0.027) & (0.035) & (0.039) & (0.028) & (0.016) & (0.010) & (0.007) \\ 0.012 & 0.013 & 0.020 & 0.080 & 0.174 & 0.372 & 0.225 & 0.047 & 0.045 & 0.011 \\ (0.008) & (0.009) & (0.010) & (0.020) & (0.029) & (0.036) & (0.030) & (0.016) & (0.016) & (0.008) \\ 0.014 & 0.013 & 0.015 & 0.023 & 0.082 & 0.180 & 0.367 & 0.211 & 0.068 & 0.028 \\ (0.010) & (0.010) & (0.010) & (0.012) & (0.022) & (0.031) & (0.039) & (0.033) & (0.020) & (0.013) \\ 0.010 & 0.010 & 0.031 & 0.013 & 0.034 & 0.089 & 0.130 & 0.342 & 0.203 & 0.138 \\ (0.010) & (0.010) & (0.017) & (0.011) & (0.018) & (0.027) & (0.034) & (0.047) & (0.040) & (0.035) \\ 0.020 & 0.043 & 0.021 & 0.023 & 0.070 & 0.076 & 0.106 & 0.173 & 0.229 & 0.240 \\ (0.020) & (0.029) & (0.019) & (0.023) & (0.038) & (0.037) & (0.041) & (0.054) & (0.061) & (0.058) \\ 0.032 & 0.032 & 0.062 & 0.034 & 0.034 & 0.042 & 0.117 & 0.171 & 0.212 & 0.265 \\ (0.030) & (0.031) & (0.040) & (0.032) & (0.030) & (0.034) & (0.057) & (0.066) & (0.070) & (0.077) \\ 0.029 & 0.055 & 0.028 & 0.026 & 0.113 & 0.059 & 0.147 & 0.078 & 0.127 & 0.340 \\ (0.027) & (0.037) & (0.027) & (0.026) & (0.050) & (0.038) & (0.058) & (0.044) & (0.054) & (0.077)\end{array}$

Invariant Distribution: $\bar{\pi}$

$\begin{array}{cccccccccc}0.032 & 0.037 & 0.035 & 0.047 & 0.096 & 0.129 & 0.161 & 0.162 & 0.138 & 0.164 \\ (0.010) & (0.011) & (0.009) & (0.010) & (0.014) & (0.015) & (0.016) & (0.019) & (0.021) & (0.027)\end{array}$

\section{Sample Size}

643 
Table A.2: Posterior Estimates: GERMAN Females (1984-1997)

\section{Initial Distribution: $\pi_{0}$}

$\begin{array}{cccccccccc}0.093 & 0.087 & 0.197 & 0.202 & 0.206 & 0.124 & 0.028 & 0.021 & 0.022 & 0.022 \\ (0.023) & (0.022) & (0.032) & (0.033) & (0.031) & (0.027) & (0.013) & (0.012) & (0.012) & (0.012)\end{array}$

Transition Matrix : P

$\begin{array}{cccccccccc}0.198 & 0.146 & 0.224 & 0.133 & 0.043 & 0.040 & 0.045 & 0.087 & 0.043 & 0.041 \\ (0.081) & (0.073) & (0.084) & (0.071) & (0.041) & (0.040) & (0.042) & (0.058) & (0.043) & (0.040) \\ 0.102 & 0.069 & 0.188 & 0.323 & 0.048 & 0.089 & 0.046 & 0.046 & 0.045 & 0.043 \\ (0.061) & (0.053) & (0.082) & (0.095) & (0.044) & (0.059) & (0.042) & (0.045) & (0.043) & (0.042) \\ 0.028 & 0.032 & 0.135 & 0.266 & 0.283 & 0.103 & 0.051 & 0.050 & 0.025 & 0.026 \\ (0.027) & (0.028) & (0.053) & (0.070) & (0.070) & (0.047) & (0.036) & (0.034) & (0.023) & (0.025) \\ 0.026 & 0.028 & 0.032 & 0.136 & 0.354 & 0.223 & 0.099 & 0.051 & 0.025 & 0.025 \\ (0.025) & (0.026) & (0.027) & (0.052) & (0.075) & (0.065) & (0.047) & (0.036) & (0.026) & (0.024) \\ 0.049 & 0.025 & 0.027 & 0.030 & 0.156 & 0.248 & 0.320 & 0.075 & 0.023 & 0.049 \\ (0.035) & (0.023) & (0.026) & (0.026) & (0.056) & (0.069) & (0.070) & (0.041) & (0.022) & (0.033) \\ 0.034 & 0.036 & 0.037 & 0.039 & 0.043 & 0.194 & 0.395 & 0.110 & 0.073 & 0.038 \\ (0.032) & (0.034) & (0.035) & (0.036) & (0.037) & (0.071) & (0.088) & (0.058) & (0.048) & (0.037) \\ 0.073 & 0.070 & 0.081 & 0.075 & 0.085 & 0.096 & 0.188 & 0.168 & 0.083 & 0.081 \\ (0.070) & (0.065) & (0.073) & (0.067) & (0.075) & (0.081) & (0.104) & (0.098) & (0.070) & (0.072) \\ 0.081 & 0.082 & 0.077 & 0.083 & 0.090 & 0.093 & 0.107 & 0.199 & 0.100 & 0.089 \\ (0.073) & (0.075) & (0.073) & (0.075) & (0.077) & (0.078) & (0.082) & (0.110) & (0.080) & (0.078) \\ 0.082 & 0.080 & 0.079 & 0.079 & 0.085 & 0.089 & 0.093 & 0.103 & 0.122 & 0.188 \\ (0.076) & (0.076) & (0.073) & (0.074) & (0.074) & (0.080) & (0.085) & (0.088) & (0.090) & (0.106) \\ 0.082 & 0.081 & 0.079 & 0.080 & 0.082 & 0.080 & 0.085 & 0.177 & 0.108 & 0.146 \\ (0.076) & (0.073) & (0.071) & (0.074) & (0.074) & (0.076) & (0.074) & (0.105) & (0.085) & (0.101)\end{array}$

Invariant Distribution: $\bar{\pi}$

$\begin{array}{cccccccccc}0.068 & 0.059 & 0.082 & 0.106 & 0.128 & 0.137 & 0.174 & 0.114 & 0.064 & 0.068 \\ (0.022) & (0.018) & (0.020) & (0.021) & (0.024) & (0.027) & (0.031) & (0.029) & (0.021) & (0.023)\end{array}$

\section{Sample Size}

